\title{
“How to Pronounce Meme." Three YouTube Channels
}

\author{
Sandy Baldwin
}

Department of English, Rochester Institute of Technology, 06-2307, Rochester, NY 14623, USA; cabgsl@rit.edu

Academic Editors: Burt Kimmelman and Philip Andrew Klobucar

Received: 17 October 2016; Accepted: 15 March 2017; Published: 21 March 2017

\begin{abstract}
Internet anomalies produce data and images beyond any authorship or source. They seem to compute and display the depths and potentials of the net "as such." Explanations and theories surround and attempt to account for anomalies, from ARGs to NSA recruiting tools. Examining three such anomalous YouTube channels, this essay does not propose a solution but rather maintains the anomalous as the constituent aesthetic and community of the net.
\end{abstract}

Keywords: YouTube; meme; rickroll; Internet anomaly; internet mystery; spam; Reddit; community

Because it amplifies our potential in so many ways, it's possible that the long-term impact of the Internet could equal that of electricity, the automobile and the telephone all rolled together.

Bill Gates, 2000 [1]

Never gonna give you up/Never gonna let you down/Never gonna run around and desert you/Never gonna make you cry/Never gonna say goodbye/Never gonna tell a lie and hurt you.

Rick Astley, 1987 [2]

Three mysterious YouTube channels. Well, two are "solved," Pronunciation Book [3] and Webdriver Torso [4]; or are they? The third, Unfavorable Semicircle [5], remains mysterious. To this list, I could add: the Markovian Parallax Denigrate; a858; Cicada 3301; and the Doge meme and its associated cryptocurrency, dogecoin, for the way it appears to be the emblem of the underlying abstraction involved in currency and an emblemization of the underlying abstraction of the net. Our investment in the channels and what they display peels away from any solution. Wasting and wasted on information is what tweaks us. I keep watching, even if I know the answers. The cool, dissipative surface of the video is its own mystery. Through the blind blot of the screen I imagine an impossible hysterical community: not the community of those of us watching screens, which indeed is a community we all share today across the heavily monitored world; but the absent community through the noir matter of the depths of the net.

A large and active community surrounds the anomalies, dedicated to solving the mysteries. This is not an FTF community; they are never face to face and in person, but "imaginary" in Benedict Anderson's sense, formed on bulletin boards, especially Reddit. They are obsessive, focused, and solitary, though committed to sharing data and discoveries. The solutions proposed for Unfavorable Semicircle by the Reddit community are typical of the response to the anomalies. The dominant theories include:

- Numbers station or clandestine communication channel.

- Automated test operations.

- Alternate Reality Game. 
- Trolling or work of abstract art.

- Recruitment puzzle (employment).

- Recruitment puzzle (secret society/cult).

- Viral marketing campaign.

- Work of a disturbed mind.

- Mundane automated script run amok [6].

All the speculations and solutions treat the channels as aesthetic phenomena, as artworks, either intentionally made or "in the wild" (i.e., as if they are the autonomous appearance of an aesthetic object). In short, the solution and destination of these channels is inseparable from the role of the art object as an example for appearances and the origins of what appears. The anomaly and the singular that appears on the net can only be exemplified and understood in terms of the art object and the tradition of aesthetics.

Each solution offers me a different sorting of the files, a different opening on the case. Each is a speculation on the internet itself. If the first situates the anomaly as a fiction that will be revealed to be an ARG, the second posits a code that will not be broken but is at least knowable as the code of some source, as emitted by some other. These initial options posit the possibility of a decryption; but other options, such as out of control scripts and test operations, presume a computational process that is more or less unknowable. In those cases, we are unable to grasp the algorithm but we derive satisfaction from knowing that an algorithm is at work. The most frequent solution involves a coded message in some form, such as a numbers station (this solution was proposed at various points for all three channels). If you search you will learn that numbers stations are shortwave stations with mysterious broadcasts, typically of a person reciting a series of numbers [7]. Numbers stations remain mysterious, but the leading theory is that these stations are used by intelligence agents as a way of transmitting a one-time pad, i.e., a single use cipher that is considered highly secure precisely because of its singularity. Numbers stations are a model of the singular and unique in the world of replicable and viral codes. A similar speculation sees the channels as products of algorithmic generation. This seemed "proven" by the revelation about Webdriver Torso (see below), yet the algorithm at work remains obscure. In these instances, the speculation relies on a belief in the productivity of algorithms, in a presumption that computational processes drive the net and produce complexity. This explanation then allows one to take the anomalous video channel, in its singular anomaly, as the outcome of the net's algorithmic production. In recognizing that we cannot read the video, we experience remotely the complexity of the net.

I am moving through sites and sorting the files on the case. There are toolkits for picking through the debris, apps to download, and protocols to initiate. Imaginary repertoires haze around the data dregs, proffering the creaky notion of "reading," as if it were ages ago, as if time travel made humanism and humanities somehow still exist. I activate reading protocols. I initiate data sorting and output, and channel it into writing. I activate de-contextualizing protocols, disconnecting linkages that lure me into depths. Looking into the files, I find reference to "deep reading" of code artifacts, I find discussion of digital hermeneutics. Each phrase is a virtual reality: from deep to reading, from digital to hermeneutics, these are fictional projects. They keep people spewing claims, training students, tossing off articles, all in the heady delusion of the fiction. I think of Tan Lin's recent work, which proposes a practice of "ambient reading." The text as a metadata sieve, the book as a medial platform, and reading as data management. Of course, this offers a new figure of reference, structure, and aesthetic response [8].

So, it is the haze of images, the residual heat of computation and distant processes, which offers me clues and links. I am on the case, sorting through the files. I need to write as if opening files, as if clicking links to sites, as if executing code. I must not write as if filling a page and creating a text. We are always writing "as if", but the imaginary at work is not a page but a code. 
I realize now that this is the case: a sharing, a mediation through untold screens; but also a massive waste, through metal and glass and plastic, a debris field utterly bereft of the codes that float in these devices. A case that is unsolved and insoluble. Is this not the situation of the net today? I am in the world and experience through my body, through this immediacy of mediation that is the world, through the phenomenological thickness set out by Maurice Merleau-Ponty, but always in the light of a screen, always my body as the terminal node of the network's illumination. I think of my crowd, the ones I hang with, and it's always in terms of the screen. Our communities only exist in this light, and their imagining and articulation occurs online. If the phone is off, if the computer is powered down, there are still other monitory screens unknown and at work. The net illuminates this inarticulate world. When will the light of our screens outshine the sun's light?

The electric light is "a medium without a message." This phrase is on my screen. I typed it into a text document sometime, when? It appears as a clue, a trail for something I started. It might be years since I began staring at this difficult statement. Yes, of course, Marshall McLuhan at the beginning of Understanding Media wrote it [9], so I can locate its origin, but not read it. I have been unable to read it for years, decades. The phrase is unreadable. It burns a hole in my eye and into my brain. Where is that electric light from? Emitted from the screen, my laptop and my iPhone and all the screens, beamed at me, filling my eyes with images. These images are without any message, nothing but medium. ${ }^{1}$ It is the same for the light hitting you and these words you're taking in and going off on: nothing but a massage for your eye without a message. In this sense, a visual sense and a sense of illumination without understanding, I am awash in "extension" without the obviousness of a mechanical arm or other cyberpunk apparatus that extension connotes. The lack accounts for the unconscious, impossible to thematize, but intense and ubiquitous mediation that bathes us, making us share in an untold space.

Yet there are messages of a sort: I am looking at this phrase on my computer screen. Here we go still further with McLuhan: these are messages without a medium, their mediation fading, nothing more than the suggestion of contexts and codes, light departed. These messages proffer readability and suggest decoding, but they are more like crumbs or crumbling fossils. Think of all those tweets and Facebook posts: they suggest readings but deliver nothing. ${ }^{2}$ The light sheds and fades from the screen and in the end there is nothing to read but the dregs of mediation itself, nothing but the faint message that mediation was at work and has now departed, faded, slipped away.

If this is the case, the clues lead into the dark, the noir depths of the net, to codes with no possible insight. More and more, I think I am blind to everything on the net. It is all cooling off, a tide receding to leave bits and parts. Decryption devices whir and click, spooling out plaintext messages. All the codes are inert, emptied. It's all tl;dr. Too long didn't read, but spelling it out defeats the purpose and increases the problem, that's already way too long and nobody would bother reading. It's just a hardened iconic cluster of characters, tl; dr, wtf. Not even vicious replies to noobs can be long enough to distract. This is the situation I'm in, but isn't this always the case? What are the senders and receivers? Where are the stoppages? Where are the files? Who opens the files, who runs the software? And, always: how do we inhabit and find ourselves at home on the net? I'm on a case, my own case as well as the case of the net. I'm cruising down those mean streets, the information highway.

Internet anomalies dissipate information, appearing without announcement, without signature or name, on sites or channels. They are computational, counting down to something and encoding cryptographic data of some message, but all messages and all somethings are hidden in the darkness of the deep web. Computation as automatic, autonomous, and autopoietic is precisely the dark materiality of writing that is at the core of the case. Contrast an email account full of spam or when you're rickrolled by a video: these viral units are characterized by memetic events, serial transmissions of cultural contents. Spam or memes are productive, flaring up with residual heat. Anomalies

Not far from this is Richard Grusin's recent formulation of "radical mediation" [10].

Or think of those proclamations of "truthiness" in an era "after the fact" or "the end of theory" in an era of big data. 
are hardened and cool, nearly cold, an end of production. Jussi Parikka and Tony D. Sampson's introduction to The Spam Book [11] declares that there are "a number of ways in which anomalies feedback into the expressive and material components of the assemblages that constitute network culture" (5). This important book does not attempt to solve or situate anomalies, but to make linkages between their singular occurrence and other forms of net phenomena. Following Parikka and Sampson, I want to know how far three YouTube channels differentiate themselves from the "new," that shiny edge of new media, where innovation or disruption is a way of extending the organization of capitalist operations. How is the anomalous a very different net phenomena from the marketing tactics of the new? How far are these anomalies anomalous?

What are the facts? Pronunciation Book and Webdriver Torso are "solved" while UnfavorableSemicircle remains mysterious. Pronunciation Book and UnfavorableSemicircle are no longer active, the first having completed its project, as it were, and the second mysteriously taken down from YouTube, without any information as to why beyond a notice of multiple Terms of Service violations. Webdriver Torso, meanwhile, continues to post hundreds of videos every day.

All three are YouTube channels. YouTube is a "video sharing website" in the way my iPhone is a telephone. I need to force myself to stop writing YouTube "video," stop fantasizing the object as a site, and open the word up to a corrosive nanosurgery. Every YouTube channel is an opening to an elsewhere that is wrapped in familiar interface features of play buttons and comments lists and links of related video. What comes through, through the opening? Music, images, old movies, horrors, beauties, faces, animals, clouds, babies, cats, blood, mountains, dead bodies, everything that can upload and play. YouTube is anything whatsoever in a wrapping. YouTube extrudes, sticking out into the interface from outside. Not videos but part objects, the tip or orifice of an exterior. All of YouTube are parts, from the most commercial plug to the most banal video of someone's cat. Our participation and satisfaction in viewing touches this exterior. It is the point or the tease, beyond the scopophilia of watching. The solution to the mystery, the discovery that Pronunciation Book is written by "dicks" and "liars," as angry readers declared after the reveal—which characterize the videos as both fiction and phallic-allows us to talk about the opening, to address and locate the stimulation the video brings. The channel brushes up and touches the opening, the channel involves a bait and switch, a deflation, a dissipation and cooling of the desire for reference, meaning, and investment. To watch the video is to absorb mediation, to share in the glow, and to close off the anomalous materials spilling through the opening. But the lie or the dick, the performance and the body part, are still there, contained but not explained. I write about these channels, I build up the case, I sort the files, but their inert mystery remains.

All three channels signify screens, through their organization as a YouTube channel and through the way specific videos simulate and reference televisual or filmic frames and imagery. At the same time, another linkage is to the iconography of the written sign. All three invoke or suggest the form of the written character and the thematics of reading and speaking. All three present characters or marks to be read, and all three-in different ways-employ a voice in the background, employ a narrator that reads. Yet none of the three allow any reading to take place, if this would mean understanding the channel and its spew of information. Once again, I underline the fact: intense speculation surrounds these channels, with large communities of active researchers and detectives, as already noted, and with arguments for their authorship and intention by aliens, spies, and artists. To watch the channels is to stage reading and writing as endless speculation on the debris field of uploaded videos.

What are the channels? Pronunciation Book uploaded 955 videos from 24 April 2010 to 24 September 2013. It gained a major cult following in the Reddit community and elsewhere, who proclaimed disappointment when the final video on the channel turned out to be a teaser leading viewers to an online game called Bear Stearns Bravo, a kind of comedy futuristic ARG. Pronunciation Book appeared to be a how-to guide to pronouncing English language words. In this, it shares with other "how to" YouTube channels with videos on How to Fight a Bear and How to Tie Your Shoes. YouTube's Content ID system algorithmically recognizes Pronunciation Book as in this genre, and 
pushes sponsored ads for other pronunciation channels, such as Pronunciation Academy and Sozo Exchange, where a typical video explains the difference between American and English pronunciation using the worlds "Trouble" and "Travel." One would expect a pronunciation "how to" channel to focus on second language learning or perhaps on commonly mispronounced words, and Pronunciation Book does include important words such as "Perhaps" and "Yield." It also includes how to pronounce "Benedict Cumberbatch," "one million dollar commercial," "Schadenfreude", and other uncommon and not especially English words. The videos show the word or phrase to be pronounced set in a black sans serif font against a white background. The effect is pedagogical and invokes infographics. A typical video, at least during the first year of posting, repeats the word to be pronounced. The first video uploaded is five seconds long on how to pronounce ASUS, and it repeats the word three times [12].

After about a year of uploads, the videos began to cover longer and more puzzling phrases, such as "help me to escape from this place" and "I'm very sorry you had to see that, Chief." The video length grew and varied, from approximately 5 seconds to over a minute with the phrases and added words alluding to some sort of narrative [13]. After three years and hundreds of videos, the uploads shortened again in length. Abruptly, the videos announced a countdown, stating in a video of the number 77 that "Something is going to happen in 77 days" and the next day a video stating "Something is going to happen in 76 days" [14]. The countdown proceeded daily. Some of the countdown videos went well beyond a countdown and the number: the number 7 is a good example, announcing "And who in death are robbed of all their worth? Something is going to happen in 7 days" [15]. This is one of the many videos suggesting an oblique but never fully articulated narrative. Jacob Bakkila, the Buzzfeed employee who turned out to be behind the project, declares on his website: "By telling a story one word or phrase at a time over the course of 42 months, and by using the most banal and innocent medium (video spam), we sought to force the reevaluation of the traditional structures and limitations of written-spoken narratives, and to challenge the definitions of signal and of noise" [16].

After the countdown, after video number one declaring "I find you in the grace of cyberspace. Something is going to happen in one days," after this was the final upload: a video of the text "horse_ebooks," which directed viewers, over a million, to horse_ebooks. Those familiar with horse_ebooks, as a spammy and humorous Twitter account combined with viral marketing tools, began to see where things were leading. The final upload did not stop there, but became a very different video: no longer the stark black on white infographic but a live actor, a spokesperson speaking of a science fiction story, which ultimately pointed to Bear Stearns Bravo, and Bear Stearns Bravo turned out to be an interactive video art game re-setting the 2008 financial crisis in the far future [17]. It proved the terminal point of Pronunciation Book, at least so far. Those liars and dicks. The fascinating countdown, the allusions to a story, all turned into an artwork vaguely about contemporary capitalism. Was the lesson simply that all things point to a commodifier? I cannot decide, as I sort through the files. The ending is abrupt and remains a mystery in its own way.

The idea of a smokescreen of fictions concealing something banal is a common way of explaining and resolving net anomalies. In this theory, the channels probably are viral marketing campaigns for a product, perhaps an artwork or game. Pronunciation Book's big reveal as a teaser for Bear Stearns Bravo is the best example. This "rickroll theory" reduces the oddness of the channel to a lure. The rickroll is an operation that extends beyond simply linking to a video of Rick Astley singing "Never Gonna Give You Up." The unexpected link to the video started as a prank on 4chan, the imageboard and net culture site. It is a widely recognized Internet phenomenon that is more than an individual meme: it functions as syntax, as an operation structuring the viewing and linking experience. The simplest explanation of the rickroll is that I click a link and the destination I think I am headed for through the link instead leads me to the Rick Astley video. The lyrics of the video, declaring "Never Gonna Give You Up" and so on, initially seem at odds with the fact that I have been redirected and mislead. Astley elaborates all the things he will never do: let you down, run around and desert you, and so on. The lyrics are a pledge of fidelity, stability, and truthfulness. Perhaps I should 
take these lyrics seriously in understanding the rickroll. This suggests that despite the redirection, the rickroll is exactly what we wanted, or at least what we get. Of course, it is not that we wanted the video of Rick Astley, but the promises of that video, which never let us down, turn out to deliver far more than whatever it was I was headed to, whatever promised site the link declared. The break between the link's statement and its performance is filled and satisfies through the rickroll.

Looking more closely: the title "Never Gonna Give You Up" voices the others' support for my existence and persistence on the net. Rick Astley, as the voice of linkages, will maintain me in ways that the promised link might not: the link might give me up, I might be lost through the link, but Rick will never do that. The rickroll smoothes and strokes our disinvestment in the part objects of the net by delivery a safety and reliability, albeit in a jokey and kitschy manner. The entropic fading of anomalous matter on the net is held off for the moment by this singing and dancing figure. I check the files again, and sure enough the video dates from 1987, two years before Tim Berners-Lee makes his earliest proposal for what becomes the web. Is Rick a reassurance from a pre-web time, where voice and image where finely coordinated in one dancing figure? I am not convinced. The status of the video as "Best Act Ever" on the net belies this [18]. No, I cannot force the rickroll into a kind of nostalgic allegory.

Are not all links rickrolls of some sort? In other words, is the rickroll a way of theorizing our disinvestment in using the net? Firstly, the link, every link, is a fragment, perhaps just an underline or a hand telling us that something is there, which proffers an outcome, offers a wholeness, promises an answer and a destination. In this sense, arriving at my intended destination is never enough. Every link I click gives me the sense that I have been given up. No link satisfies, but every link teases and pleases. In this way, Rick is far more reassuring. He closes the opening of the link, he maintains me, never gives me up.

The Webdriver Torso YouTube channel uploaded over 490,000 videos since September 2013, with hundreds of new videos continually added daily up to the present. Almost all of the videos consist of slideshow sequences. The number varies, but is typically around ten slides. The length of video varies greatly, but they are typically around eleven seconds. The slides are colored and can be described as Mondrian-like: rectangles of red and blue and white. Initially, the names of the videos began with the word "aqua" followed by random characters; eventually videos were posted with names beginning with "tmp" followed by random characters. Several other apparently related channels post similar videos: Webdriver YPP, Ekaterina Basic, and Webdriver IVPE. Almost all of the videos consist of slides of colored rectangles, but there are notable, well-documented exceptions. The video labeled 0014 shows an image of the Eiffel Tower at night, the illumination invoking the colored slides on the typical videos [19]. This five-second video pans from the tower to a laptop held by the videographer, before ending. And then there is the video tmpRkR185 [20], which moves from a typical slide show to a silhouette of Rick Astley dancing. Clearly we have been rickrolled, but here the satisfaction and destination are unsettling, which is reinforced by the fact that we get only the outline of Astley, a ghostly rickroll formatted against the familiar Webdriver Torso colors. Why do I find it unsettling, this ghostly Rick? Is it that unlike Pronunciation Book? Is it that, somehow, Webdriver Torso is far less easy to enclose and contain?

Like Pronunciation Book, a large community engaged in wide-ranging speculation and computational analysis about Webdriver Torso: was it aliens? An ARG? A numbers station? After a year of posting, the channel was "solved" in June 2014, when some sleuthing by dedicated Reddit members led to the announcement that the channel was used by Google, the owner of YouTube, to test video quality. As a kind of proof, if you type Webdriver Torso into Google Search, the icon in the upper left is replaced by typical video image colored squares. Finally, when pressed, Google released the statement: "We're never gonna give you uploading that's slow or loses video quality, and we're never gonna let you down by playing YouTube in poor video quality. That's why we're always running tests like Webdriver Torso." For many, there is something odd and false about this rickroll revelation: why 
use these algorithmically generated slides to test video quality? Was Webdriver Torso a kind of cover for some other operation? After all, why the secrecy?

The third channel, Unfavorable Semicircle remains enigmatic, and its suggestion of writing is hardest to imagine reading. It eludes the imaginary, offering no clearly connoted images, offering at most a constellation of dots and marks. Starting on 5 April 2015, Unfavorable Semicircle published hundreds of videos daily, very much like Webdriver Torso. The images were vague and hazy. On 25 February 2016, the channel was abruptly shut down after publishing over 96,000 videos, with statements that it violated YouTube's Terms of Service. This is despite the fact that none of the videos contain any ostensible content, beyond the cloudy images. The YouTube shutdown seemed to rule out that the channel was another in-house video quality testing site like Webdriver Torso. The Reddit investigators found a trail through garbled text in YouTube comments, which led to a Twitter feed and a new YouTube channel called UnfavorableSemi. New uploads began on this channel.

The videos, both on the old and new channels, tended to be short, several seconds in length; but one lasts almost seven hours. As noted, the images are more abstract than Webdriver Torso, appearing like a miasma or cloud, or at times very dark with flashes of light. Many include a deep voice uttering words that cannot be understood. Others contain long passages of seemingly white noise or audio static. The pattern and the voice appear random, noisy, and unreadable. Whereas Pronunciation Book couples voice and image into a simulation of a how to pronounce video, albeit with often-mysterious content; here voice and image are decoupled and resist connotation. There is not enough of either to suggest purpose or message. At best there is the suggestion of some ordered image and the suggestion of a voice.

Are these channels the result of some deeper source in the net? What if anomalies are structural results of the net itself? That is: the writing of some level of the net so unattainable that the writing can only be read as a mystery. This is the common speculation that the channels result from unknown and unknowable computational or conspiratorial operations in the depths of the web. In these theories, the origins are not traceable, but remain only traces of these depths. The only possible decryption is a continuation of the mystery. In such speculations, the community deciphering the three channels references the deep web or dark net, as the ultimate and unknowable source of the uploads. The anomalous appearance of writing-like marks is read as written from these depths.

Deep and dark: networkers distinguish the deep web, the dark net or dark nets, and the dark web. The first is that part of the web that is not indexed by search engines. This would include content that is hidden for proprietary or private reasons, remaining blocked by robots.txt or hidden behind firewalls. It would also include dynamic or contextual content, webpages delivered only on the fly. It would also include derelict pages, unlinked or lost. Estimates are that the deep web is hundreds of times larger than the surface web, i.e., the web we all commonly use. Evidence shows that discussion of the "invisible web" and similar formulations appeared as early as 1994; that is, right at the origin of the web. The term "deep web" allows a gesture towards the vastness of the web beyond what can be shown or accessed. Deepness is spatial: a certain amount is visible, indexable; the rest hidden like an iceberg. But the term is also temporal: only a certain aspect and part of the web is available at any moment, and dynamic content means that the web is constantly changing. In this sense, the deep web's size cannot be measured: it is built and destroyed every moment; its depth is the depth of transitory existence and the flicker of the screen. In this sense, to read deeply is a protocol that engages with and seeks to narrate the ephemeral and temporal quality of the web.

The dark net or dark nets, by contrast, are particular networks overlaid on the net for purposes of privacy or concealment. Such dark nets create "deep" content. The point of a dark net is privacy, with many possible motivations. Users typically access material with TOR and other specialized software. Finally, the dark web is all the sites and content on dark nets. Why the distinction? The deep web is by definition larger than the dark web, since the former includes matter that is "clear" or accessible but not indexible, because of its dynamic nature or other features. The dark web, by contrast, is entirely 
characterized by avoiding exposure. It is associated with criminal activity, most famously the Silk Road case.

Certainly there are depths to the web, but these speculations also function as suppositions or techniques for deepening or "making deep." The easy confusion of deep and dark, slipping and obscured even in the discussions by those supposedly in the know, points to the way these terms describe both particular layers to the net and structural effects of some elusive net "as a whole." Surface phenomena can be articulated with deep and dark origin. The result is an autoproductive or autopoietic theory of the net. Deep and dark refer to this ground of self-production. The terms figure the net as a whole, and its ongoing existence. The suggestion of writing in the anomalous channels-the invocation of readable marks, explicit in Pronunciation Books' infographics, connoted in Webdriver Torso's series of colored squares-appears to inscribe messages from the web itself without allowing us to read them. The non-reading and non-comprehension is the mediation, without message, of and by the depths. In this way, we participate in the web "as a whole" and "as such."

The very thought of an internet mystery, of an anomalous YouTube channel, is a hopeful speculation about the depths of the net. But what is not mysterious and anomalous on the net? Why care about three odd YouTube channels? The Internet Protocol, the IP in TC/IP, supports everything else on the net, and in truth it supports nothing. IP is the basis of the internet, but does not guarantee delivery, does not guarantee accuracy, does not guarantee preventing duplication, does not guarantee preventing misdelivery, guarantees nothing except end-to-end transmission of packets. The most basic protocol of the internet is unreliable. The web is structurally built around anomaly.

IP allows null routes or network routes that go nowhere, without destination. This can also be called a "black hole route." Packets of data are "dropped." Not only do they vanish, but the sender is never informed, never told that the packets were discarded. A null route may be used purposefully as a kind of firewall or filter. On the surface, where we deal with URLs and emails and the like, every communication on the net is destined, sent to an origin. Everything is an address. Yet there is no destination in the interior, only transport; there are no addresses, only mobility. The black hole is simply the possible outcome of this fact: a network route with no destination is a way of discarding information and making communications vanish. Data sent down a null route without intention, without purposefully sending it off, is lost without a trace. The sender does not even know. The route and the resulting black hole can only be recognized by mapping and monitoring the traffic around it, as if mapping a gravitational field. Are the anomalous YouTube channels like black holes, in the way they attract speculation and community, in the way they are sites for our desires?

The expenditure of effort to solve the anomalies by a determined and eager community is staggering. For example, the Reddit community on the question of Unfavorable Semicircle will scan the code of thumbnail jpgs taken from the video and scan for patterns, or they will scan the audio tracks of the video using spectrum analysis software, such as TrueRTA or SPEK. In either case, they search for patterns using a base64 binary to text encoding scheme, allowing them to look at long strings of ASCII decrypted from audio and visual information. Are there messages converted to binary code and then hidden steganographically in the images or across the audio spectrum? The cloudy images and the barely-heard and not understood voice on the Unforgiveable Semicircle videos is analyzed into long strings, which may contain encrypted data such as the following:

$$
\begin{aligned}
& \text { iVBORw0KGgoAAAANSUhEUgAAADIAAAAyCAIAAACRXR/mAAAA4EIEQVRYh } \\
& \text { e3WO47DMAxFUX7sXXqFmWK250IkUxBxMZgUTxDgIHi3dCEcS5Rh/Xn8yudldwP+j } \\
& \text { ywkspDIQiILiSwkspDIQlrGighVFZHzPOtVRNzMMrMxhru7u6pmZj+8mXUcR1WJS } \\
& \text { GZGRFW5e+Mm0lU/zVXVh3htlaqOMbZtm1ht5cj3bolIRJhZVe37PrfUzKu865qnPj53v+ } \\
& \text { 4B2sqR73nPTDPLzKqaHvllu5WZvVvu3vMuIn0372Sp6h/BhZvo27/yayMLiSwkspDIQi } \\
& \text { ILiSwkspDIQiILiSwkspA+lPUEckRfhTTivtYAAAAASUVORK5CYII= [21]. }
\end{aligned}
$$

I am fascinated by this determination to find messages in the buzzing audio and floating imagery. The sound and light offer me little beyond more mystery in their code. Similar effort at decryption is 
expended on net anomalies such as Cicada 1301 and A858DE45F56D9BC9. ${ }^{3}$ The former was a series of cryptographic and other puzzles and alternative reality games posted on four separate occasions to recruit codebreakers. The purpose of the recruitment is still mysterious as is the organization in the background [22]. The latter, also known as a858, has posted random strings to Reddit daily for more than five years. The following is typical:

8fd6235d9880883c8fac931007b4cf41 ab0b211c3d16b2c52c29b1bf02f1ef57 767c20e9b6cf47160af573ae3c4fd87a 17e6ae34537bfdc8480bbbcf4dee47f2 b473b392d85dee211b8a4c4695327ed8 ab237d9db363d13882a787e307e2c490 27ecd0c282636655e4768c531e17aa9f c69b05160a2e974bec9e55650b7ea381 ef971c0d87b43c5c3501157e880dd64e 96726805f79a48a51b8e8c3d5e3f6131 244a4a71837f64534a3dff729aaa1174 12d6172a73dd8c7ad2766ba812499a9a 4511dc20d0f200c53aba3d6454861bcb d2634749ba1eb0046eaee609984c4e44 78bad7b17276dab8439d7a399272b1c1 1c0e467fcde4c46ca0ab49be795f9d4b f469abd0a3dd6dd56499f0302911fc62 4f77dddc437df56b220a9395d885c055 f94035e5b71d3f652b9adf59e0985c5f 0bd01fbd6374e4f4aaca1718b98a17e7 2b071fda6d6d758ae175ab31338e148a be918f793f55475a0f853e980d60ba78 a9c5d4cb33d90295999c73a2c129cf91 43f24cee6ad4c0a4d56813986eb824f8 9ead2d68d1258d2f053421efdb99baab 3c71dbfebbf287cb69b840f5bbf40b1c e9040aa8265d3d5cf09d3cefa11d14ef cea4eeff428976b6707c388bd4ab669f f18165015019e686fe64a34007151704 61d1d7178a6c20034e75018c0281e70f 737976e1f7c7c6e76fd6fe808e6d0902 0c1874af897012464daf79c038b6a25f c642ffd475dc555f6328786826896da4 ebc398759f7e405b587962e9bc5bb7b6 0712010890dd210c60103a85ee163de9 2b6ee82b4f6194d1d27f45cce04459d7 49baf75be1cbb879e9c06deb80a1efef eee12ebe8bfd8c72bc7377b6631d8b9c b02d84710474797a481ed53b1ae2ed5b 8c8bdc63725e1ff72cdd32300133a5f8 5cfa4ec8a6f35ce1654ce69fe630821a dbbf52ba8bdf77fefdbb9f817651a0e5 8ebb0a8bb9856b4cdcef4daca4fffb61 271a0055a068dcc792f40e4a5aa9cdab 2993994159ddb2314b93646f4e9ea603 231d3d66ad5c4957 [23]. 1904edc569c977d2a743633713ecf677 31491e9ae18f010090da19f7481cc398 2e30a5f64761a46a0af133b0a63e5f48 1a3af18dc0962346cd0de78171bcb50d af7938f9d6f6eb5f9169c95fb9a492e2 $8 \mathrm{bc} 024 \mathrm{~d} 4 \mathrm{fbbf5b} 8 \mathrm{ac} 864 \mathrm{caa} 576328 \mathrm{dbb}$ 48165cb73c1aa6619c1d38b9bb7a14e0 570108288f10a148bb9543763023bfc5 6eb298a15405e5baa2b7909edf56adc3 db900a2a8e9665bb26d64978f9f812b6 28760d6a7b2a1c4dc9662d2cbdff85ee 7f3af6292b029690c047bdfc54a76ca4 07777d27d3013a06b540d1fe8d61489b 746297cddbe7de7d0067942f145a697e 76def36defabf902ce5de5fdbd0d156d 85f4ad0891ed9e9612b34412c4de44b1 deb30407ae79e6754a73e3321239e492 fa193de899dd6a2c6918233d6b15be2a b15e8774147cee659c1260486ffd37c0 e0dacaec723211c41e2e16a567e3f247 ff2ad7dcfd885db277c99912ea350fee 700c957c5af2a0971e25fd233f667044 3e6a92f0dd1a6ed9db5d3c19c5abe0fd 88f0dbfc097e31ecc983ad32a7d3361a 7db24b2f80bb17b609407f2ccf0315f4 b0d3abff77bdb03f1b01d03f702ed8a2 d56f6193218f9506ace028c1915962f5 aa38c10d7fa1fc0b3ed11f5720e951ce cd9a3a1f1f83293cf1ff274307fe0196 d5050f54afd7359a74172178a4693009 536287825028dfcf5f1a07a255700da4 d01754e34e1a9e025600c2713a16e624 b8ff96b81277a8db145b5ed2b0a51c7d 2c2870d98643d5dbc751b1eaefc542d2 7baee76605ff4e17eb96e65addfbf6d0 05cea3dc18bb8b80f5a631785e5dc6d2 d30f9538bac2f4aecbf779e3f110ca56 3e619816f2aa831e9d4c8037bc143d96 26327b622794eee5440966efa236db0c 7b8d3900229733eaeb000e869f6026ce e164560aa8607458fd744d7403a702e7 614cde4a7ed76fe78aafca0515ee398d 583122a40ac4bf60167f860b759d22ac

3 Work in progress discusses these anomalies. 
The Reddit community will carefully, excruciatingly, exquisitely, pick through this code, seeking a message. Let's face it: no message will be found, because no message will suffice. For me, sorting through these files, I recognize that the message is not the point; that these are anomalies without message. Or rather, more clearly: no end of messages that are not read, are without mediation, are simply marks. The effort is sacrificial and endless. These are practices of deepening and supplication to the net.

The case is that such writing assembles data that trickles rather than links; tepid information that leads nowhere. At best, my writing offers an image of a site, without a thesis or an argument. Clarity and conclusions are outmoded tech. What I hope for: a writing that attains the status of a datagram, spewed on the wires. Every sentence is a mnemotechnics of oblivion, deleting the data processed, demediating technical features into ambient screen glow. Disinvestment: not the psychoanalytic of investment (besetzung) into the image, not a cathecting of the other through the image surface-all of which are precisely the logic of exemplarity, as if intense reading of an image would lead me towards the otherness I am describing-but instead a passive, neutralized state of entropic chilling when unknown investments are already lost. In such a state of disinvestment, I describe and speculate, but end nowhere. Investment poured imaginary and psychic contents into the thing, pathologizing the site in terms of the subject. But the subject online can only invent hysterical pathologies in a condition of disinvestment, with no content to work through. The net's links and sites are deadened and cauterized of content, every link a rickroll, every text and image wasted, adrift, uncertain.

My attention is back on the efforts of the community to solve the anomalies. Not simply the imaginary is at work in this communal effort: this is a hysterical community, articulated and able to communicate in relation to the excess and mystery of the anomalies. The hysteria is a speaking of the community through this exterior. The community forms through shared speculation on the impossibility of the mystery. This impossible relation is tied to computation, to revelation through computation, and through writing discovered in the computational process. An unreadable and impossible writing that never turns into a message, and that turns away from any message. This is a deeply nonhuman community, committed to what is latent but never read in the web. The hysterical community around messages without mediation re-tells the dreams of the nonhuman that lies deep in its house, in the net, that lies and waits.

Conflicts of Interest: The author declares no conflict of interest.

\section{References}

1. Gates, Bill. "Shaping the Internet Age." Microsoft News Center, December 2000. Available online: https: / /news.microsoft.com/2000/12/01/shaping-the-internet-age/\#sm.0000v7ehvpdilfd5116lb8z4hoiq4\# 2f4JqJDRE3krltcA.97 (accessed on 20 March 2017).

2. Astley, Rick. “Never Gonna Give You Up." Whenever You Need Somebody. Vinyl and CD. Directed by Stock Aitken Waterman. New York: RCA Records, 1987.

3. YouTube. "Pronunciation Book." Available online: https://www.youtube.com/user/pronunciationbook (accessed on 20 March 2017).

4. YouTube. "Webdriver Torso." Available online: https://www.youtube.com/channel/ UCsLiV4WJfkTEHH0b9PmRklw (accessed on 20 March 2017).

5. YouTube. “Unforgivable Semicircle." Available online: https://www.youtube.com/channel/ UCRMFZ2kVS_pMn3MCO-t4o-w (accessed on 20 March 2017).

6. UnfavorableSemicircle. "Discussion: Should there be a master list of prevailing theories? " Available online: https:/ /www.reddit.com/r/UnfavorableSemicircle/comments/47govn/should_there_ be_a_master_list_of_prevailing/ (accessed on 20 March 2017).

7. Wikipedia. "Numbers station." Available online: https://en.wikipedia.org/wiki/Numbers_station (accessed on 20 March 2017).

8. Genusa, Angela. “A Book is Technology: An Interview with Tan Lin.” 24 October 2012. Available online: http:/ /rhizome.org/editorial/2012/oct/24/interview-tan-lin/ (accessed on 20 March 2017). 
9. McLuhan, Marshall. Understanding Media: The Extensions of Man. Cambridge: The MIT Press, 1994.

10. Grusin, Richard. “Radical Mediation." Critical Inquiry 42 (2015): 124-48. [CrossRef]

11. Parikka, Jussi, and Tony D. Sampson, eds. The Spam Book: On Viruses, Porn, and Other Anomalies from the Dark Side of Digital Culture. New York: Hampton Press, 2009.

12. YouTube. "How to Pronounce ASUS." 14 April 2010. Available online: https://www.youtube.com/watch? v=nSk4nDTnFgw (accessed on 20 March 2017).

13. YouTube. "How to Pronounce Nicole." 10 November 2012. Available online: https://www.youtube.com/ watch?v=_emnMO7-nPo (accessed on 20 March 2017).

14. YouTube. “How to Pronounce 77." 23 September 2013. Available online: https://www.youtube.com/watch? v=ouiDKPYV_zI (accessed on 20 March 2017).

15. YouTube. "How to Pronounce 7." 17 September 2013. Available online: https://www.youtube.com/watch? $\mathrm{v}=$ PmWRINdO5y4 (accessed on 20 March 2017).

16. Jacob Bakkila. Available online: http://jacobbakkila.com/ (accessed on 20 March 2017).

17. Bear Stearns Bravo. Available online: http:/ /www.bearstearnsbravo.com/ (accessed on 20 March 2017).

18. BBC News. "Astley shortlisted for MTV award." Available online: http://news.bbc.co.uk/2/hi/ entertainment/7646807.stm (accessed on 20 March 2017).

19. YouTube. “00014.” Available online: https://www.youtube.com/watch?v=SKvIyDB5FRU (accessed on 20 March 2017).

20. YouTube. "tmpRkRL85." Available online: https://www.youtube.com/watch?v=klqi_h9FElc (accessed on 20 March 2017).

21. UnfavorableSemicircle. "The Successful decoding was probably a hoax." Available online: https:/ / www.reddit.com/r/UnfavorableSemicircle/comments/4bx1z7/the_successful_decoding_ was_probably_a_hoax/ (accessed on 20 March 2017).

22. Cicadianism. Available online: http:/ / cicada3301.org/ (accessed on 20 March 2017).

23. REDDIT. Available online: https://www.reddit.com/r/A858DE45F56D9BC9/ (accessed on 20 March 2017).

(C) 2017 by the author. Licensee MDPI, Basel, Switzerland. This article is an open access article distributed under the terms and conditions of the Creative Commons Attribution (CC BY) license (http:/ / creativecommons.org/licenses/by/4.0/). 